\title{
Light sterile neutrino and low scale left-right symmetry in a D-brane inspired $S U(4)_{C} \times S U(2)_{L} \times S U(2)_{R}$ model
}

\author{
George K. Leontaris ${ }^{1, *}$ and Qaisar Shafi ${ }^{2, \dagger}$ \\ ${ }^{1}$ Physics Department, Theory Division, Ioannina University, GR-45110 Ioannina, Greece \\ ${ }^{2}$ Bartol Research Institute, Department of Physics and Astronomy, University of Delaware, \\ DE 19716 Newark, New Jersey, USA
}

(Received 5 December 2018; published 20 February 2019)

\begin{abstract}
Motivated by the ongoing searches for new physics at the LHC, we explore the low energy consequences of a D-brane inspired $S U(4)_{C} \times S U(2)_{L} \times S U(2)_{R}$ (4-2-2) model. The Higgs sector consists of an $S U(4)$ adjoint, a pair $H+\bar{H}$ in $(4,1,2)+(\overline{4}, 1,2)$, and a bidoublet field in $h(1,2,2)$. With the $S U(4)$ adjoint, the symmetry breaks to a left-right symmetric $S U(3)_{C} \times U(1)_{B-L} \times S U(2)_{L} \times S U(2)_{R}$ model. A missing partner mechanism protects the $S U(2)_{R}$ Higgs doublets in $H, \bar{H}$, which subsequently break this latter symmetry at around to $10^{4}-10^{6} \mathrm{GeV}$ to the Standard Model. An inverse seesaw mechanism generates masses for the observed neutrinos and also yields a $\mathrm{keV}$ sterile neutrino that can play the role of dark matter if its mass lies in the $\mathrm{keV}$ range. Other phenomenological implications, including proton decay, are briefly discussed.
\end{abstract}

DOI: 10.1103/PhysRevD.99.035032

\section{INTRODUCTION}

Spontaneously broken left-right symmetry and the idea of quark-lepton unification are nicely encapsulated in the $S U(4)_{C} \times S U(2)_{L} \times S U(2)_{R}$ (4-2-2) gauge symmetry proposed by Pati and Salam (PS) [1]. With gauge bosons that can mediate proton decay absent, the 4-2-2 symmetry may well be broken at scales that are orders of magnitude lower than the usual grand unified scale $M_{\mathrm{GUT}} \simeq 2 \times 10^{16} \mathrm{GeV}$. A lower bound on the 4-2-2 breaking scale, of around $100 \mathrm{TeV}$, arises in the simplest model from the nonobservation of the decay $K \rightarrow \mu+e$ mediated by some of the gauge bosons in $S U(4)_{C}$. The experimental constraints on the left-right gauge symmetry breaking scale are significantly milder [2,3], and indeed, this symmetry may be broken at a scale that may be accessible either at the LHC or its upgrades.

In this paper, we propose to explore the low energy consequences of a 4-2-2 model derived from an intersecting D-brane framework. As is well known, the 4-2-2 model realizes quark-lepton unification in a natural way. Several other attributes of this symmetry [4-7] are the incorporation of the right-handed neutrino in the spectrum, the

\footnotetext{
*leonta@uoi.gr

†shafi@bartol.udel.edu
}

Published by the American Physical Society under the terms of the Creative Commons Attribution 4.0 International license. Further distribution of this work must maintain attribution to the author(s) and the published article's title, journal citation, and DOI. Funded by SCOAP ${ }^{3}$. absence of gauge bosons mediating fast proton decay, etc. ${ }^{1}$ In the last few years, there has been growing theoretical and experimental interest in the theory of fundamental interactions in the $\mathrm{TeV}$ region. We believe that the 4-2-2 model incorporates all of the ingredients to interpret possible related findings, and therefore in this note, we would like to examine low scale symmetry breaking patterns and explore possible predictions. Indeed, the present experimental bounds on scalar superpartners are close to the $\mathrm{TeV}$ scale, while searches for new gauge bosons have put lower limits at a few TeV. Supersymmetric scalar masses of the minimal supersymmetric Standard Model (SM) states are definitely related to supersymmetry breaking, while the existence of new gauge bosons are naturally associated with some new symmetry breaking scale. A natural candidate of such, a new gauge symmetry, not far above the electroweak (EW) scale, is $S U(2)_{R}$, so that above this breaking scale, the model is left-right symmetric. Moreover, there is a good chance that the associated gauge boson with a relatively low $S U(2)_{R}$ breaking scale leaves its signature in experiments through new interactions, which may be identified in future Diboson searches [9]. The left-right symmetric model is naturally embedded in a quark-lepton unified (4-2-2) symmetry, which admits an interesting string realization in the context of heterotic superstrings, the intersecting D-brane scenarios, as well as in F-theory [10-13]. However, although the 4-2-2 symmetry unifies

\footnotetext{
${ }^{1}$ For a recent review see Ref. [8].
} 
quarks and leptons, its symmetry structure consists of a product of non-Abelian factors and, as such, does not necessarily imply unification of the gauge couplings. Given this fact, from the above string theory realizations, the intersecting D-brane set up appears to be the most natural scenario. Indeed, within this context, each group factor is associated with a different brane stack with its own gauge coupling strength, therefore the 4-2-2 model is naturally motivated in an intersecting D-brane framework. Moreover, the tight connection between the unification scale and the Planck scale $M_{\text {Planck }}$ is no longer mandatory, and the symmetry breaking scale can be substantially lower than $M_{\text {Planck }}$.

In this paper, we investigate the realization of a two-step symmetry breaking scenario of the PS model built in the context of open string theories and D-branes. At the first stage, the PS symmetry breaks at $M_{\mathrm{GUT}}$ to the left-right (LR) symmetric model with the use of the adjoint Higgs. At a second scale $M_{R} \ll M_{\mathrm{GUT}}$, a pair of right-handed Higgs doublets triggers the breaking of the LR-symmetry down to the Standard Model one.

The layout of the paper is as follows: In Sec. II we discuss the D-brane framework for the 4-2-2 model and present the particle spectrum in Table I. A variety of new fields appear that are absent in the standard field theory constructions. They include a SM sterile neutrino that turns out to be a plausible dark matter candidate. In Sec. III we consider the two-step breaking of 4-2-2 to the $\mathrm{SM}$. We exploit here a triplet-singlet splitting mechanism, which is analogous to the well-known doublet-triplet mechanism of $S U(5)$. The left-right symmetry breaking

TABLE I. Minimal Spectrum and the corresponding quantum numbers that emerge in a D-brane configuration with $U(4)_{C} \times$ $U(2)_{L} \times U(2)_{R}$ gauge symmetry.

\begin{tabular}{lcrrr}
\hline \hline Intersection & $S U(4)_{C} \times S U(2)_{L} \times S U(2)_{R}$ & $Q_{C}$ & $Q_{2 L}$ & $Q_{2 R}$ \\
\hline$a b$ & $3 \times F_{L}(4, \overline{2}, 1)$ & 1 & -1 & 0 \\
$a c$ & $3 \times \bar{F}_{R}(\overline{4}, 1,2)$ & -1 & 0 & 1 \\
$a c^{*}$ & $\bar{H}(\overline{4}, 1, \overline{2})$ & -1 & 0 & -1 \\
& $H(4,1,2)$ & 1 & 0 & 1 \\
$a a^{*}$ & $S_{10}^{ \pm}(10,1,1)$ & \pm 2 & 0 & 0 \\
& $D_{6}^{ \pm}(6,1,1)$ & \pm 2 & 0 & 0 \\
$c c^{*}$ & $\Delta_{R}(1,1,3)$ & 0 & 0 & \pm 2 \\
& $(N, \bar{N})-$ singlets & 0 & 0 & \pm 2 \\
$b b^{*}$ & $\Delta_{L}(1,3,1)$ & 0 & \pm 2 & 0 \\
& $\left(\nu_{s}, \bar{\nu}_{s}\right)-$ singlets & 0 & \pm 2 & 0 \\
$b c$ & $h(1,2, \overline{2})$ & 0 & 1 & -1 \\
& $\bar{h}(1, \overline{2}, 2)$ & 0 & -1 & 1 \\
$b c^{*}$ & $h^{\prime}(1,2,2)$ & 0 & 1 & 1 \\
& $\bar{h}^{\prime}(1, \overline{2}, \overline{2})$ & 0 & -1 & -1 \\
$a a$ & $\Sigma(15,1,1)$ & 0 & 0 & 0 \\
$b b$ & $\Sigma_{3_{L}}(1,3,1)$ & 0 & 0 & 0 \\
$c c$ & $\Sigma_{3_{R}}(1,1, \overline{1})$ & 0 & 0 & 0 \\
\hline \hline
\end{tabular}

scale lies in the $\mathrm{TeV}$ range, which may be found at the LHC or its future upgrades. Section IV contains a discussion of neutrino masses that includes the inverse seesaw mechanism and a sterile $\mathrm{keV}$ mass neutrino. A brief discussion in this section of proton decay shows that it is adequately suppressed in this class of models. Our summary and conclusions are presented in Sec. V.

\section{4-2-2 SPECTRUM FROM INTERSECTING D-BRANES}

In this section, we will present the basic features of an open string realization of the 4-2-2 gauge symmetry. In general, there are several methods to construct 4-2-2 vacua in string theory, such as Calabi-Yau compactification, Type IIA string theory, interacting conformal field theory constructions, and also Gepner constructions. For the purposes of this work, we find it convenient to represent these models in terms of intersecting D-brane configurations, which is the appropriate description for type IIA string theory.

The low energy phenomenology of the 4-2-2 symmetry built in the framework of intersecting D-brane scenarios differs in many respects from the corresponding model that admits an $S O(10)$ embedding [7]. The main reason is that intersecting D-brane constructions yield an $U(4) \times U(2) \times U(2)$ gauge group, which is the 4-2-2 symmetry augmented by three $U(1)$ factors. Indeed, recalling that $U(n) \simeq S U(n) \times U(1) / Z_{n}$ the final gauge symmetry of the D-brane version of the effective field theory model is

$S U(4)_{C} \times S U(2)_{L} \times S U(2)_{R} \times U(1)_{C} \times U(1)_{L} \times U(1)_{R}$.

The intersecting D-brane setup associated with the above symmetry is depicted in Fig. 1. Each gauge group factor $U(n)$ is associated with a set of $n$ parallel, almost coincident, D-branes, while the various massless states are represented by open strings attached on the various sets of branes in the appropriate configurations. This set up gives rise to bifundamental representations that accommodate the particle spectrum of the model. The most general picture of the available representations is as follows: (i) Open strings connecting two brane stacks give rise to bifundamental representations with respect to the corresponding gauge groups, (ii) open strings stretched between a D-brane and its image transform in the antisymmetric or symmetric representation of the gauge group, and (iii) for each group factor, we should include the adjoint representation, which arises from open strings stretched between branes in the same stack. 


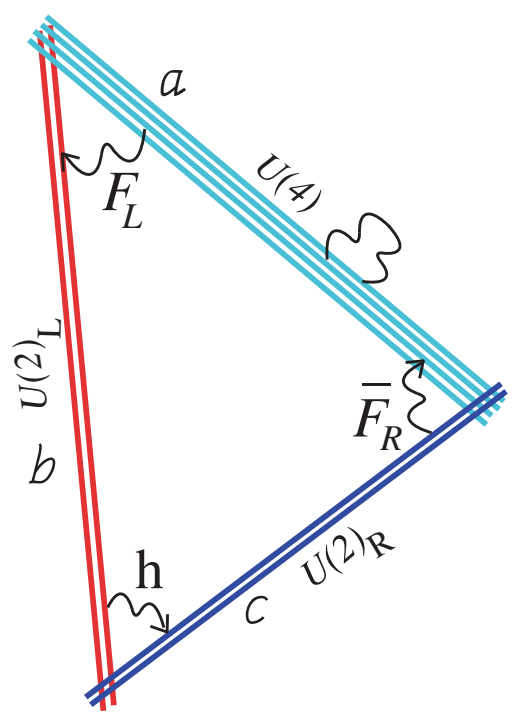

FIG. 1. Intersecting D-brane configuration for the 4-2-2 symmetry. The letters $a, b, c$, respectively, stand for the $U(4)_{C}$, $U(2)_{L}$, and $U(2)_{R}$ brane stacks. Under orientifold planes there are the mirror branes (not shown in this figure) denoted with $a^{*}$, $b^{*}, c^{*}$ in the main text.

To underline the salient features of the D-brane derived 4-2-2 models, in Table I we present a minimal set of fields obtained in the context of a D-brane setup, which are required for the realization of a viable effective model. There are states originating from string vibrating at the intersections $a b, b c, c a$ of the three D-brane stacks $a, b, c$. There are also states emerging from strings with ends attached to the mirror branes $a^{*}, b^{*}, c^{*}$ (not shown in the figure). The observed chirality of the spectrum can be adjusted from appropriate string boundary conditions but, in general, the spectrum may contain additional vectorlike pairs.

As expected, all massless states associated with the open string spectrum, are charged under the extra $U(1)$ factors. Indeed, returning to the content of Table I, we note that the last three entries show the charges of the representations under the three Abelian factors $U(1)_{C}, U(1)_{L}, U(1)_{R}$. It is remarkable that the Abelian factor $U(1)_{C}$ plays a central role in defining the baryon and lepton quantum numbers. Indeed, if we consider the breaking

$U(4)_{C} \rightarrow S U(4)_{C} \times U(1)_{C} \rightarrow S U(3) \times U(1)_{B-L} \times U(1)_{C}$, we find out that they are defined in terms of the linear combinations [11]

$$
\begin{gathered}
U(1)_{\mathcal{B}}=\frac{1}{4}\left(U(1)_{C}+U(1)_{B-L}\right), \\
U(1)_{\mathcal{L}}=\frac{1}{4}\left(U(1)_{C}-3 U(1)_{B-L}\right) .
\end{gathered}
$$

We note that in the context of the Standard Model, these are global symmetries that are anomalous. In the framework of intersecting D-brane constructions, the $U(1)_{C, L, R}$ symmetries are also anomalous, and only particular combinations are anomaly free. It transpires that these $U(1)$ anomalies are cancelled by the generalized GreenSchwarz mechanism (through couplings between the RR two form field and the corresponding field strength, see e.g., Ref. [14]), which induces masses for the corresponding gauge bosons. Yet, at the perturbative level, these remain as global symmetries that prevent rapid baryon and lepton number violating processes.

To be more precise, in the presence of such $U(1)$ symmetries, the Fayet-Iliopoulos D-terms are expected to appear with magnitudes of the order of the compactification scale. These must be canceled by contributions from scalar fields, carrying charges under these $U(1)$ 's, and developing vacuum expectation values (VEVs) of the order of the compactification scale. Since the magnitude of these VEVs also determines the breaking scale, the anomalous $U(1)$ symmetries are expected to break at the compactification scale too. The solution of these issues is related to the specific string construction one considers, and it is of particular importance in the subsequent analysis since it is decisive in shaping the effective field theory model and its low energy predictions.

Therefore, to make the above arguments more transparent, we present the exact spectra for two specific cases chosen from a variety of models presented in Ref. [15], where the multiplicities of the representations in Eq. (5) are derived in the context of Gepner constructions $[16,17]$. The spectra of the two models, dubbed $\mathbf{A}$ and $\mathbf{B}$ here, appear in Eq (5), where the relevant sectors are indicated in the first column of each model. The second and third columns show the multiplicities and the chiralities of the corresponding representations, which are designated in the last column of each model. 
Model A

\begin{tabular}{||lrrl||}
\hline Sector & $\#$ & chirality & Spectrum \\
\hline \hline$a b$ & 6 & 2 & $4 F_{L}+2 \bar{F}_{L}$ \\
\hline$a b^{*}$ & 5 & 1 & $3 F_{L}+2 \bar{F}_{L}$ \\
\hline$a c$ & 2 & -2 & $2 \bar{F}_{R}$ \\
\hline$a c^{*}$ & 3 & -1 & $\bar{F}_{R}+H+\bar{H}$ \\
\hline$a a^{*}$ & 4 & 0 & $2\left(S_{10}+\bar{S}_{10}\right)$ \\
\hline$b b^{*}$ & 5 & 1 & $3 \nu_{s}+2 \bar{\nu}_{s}$ \\
& 4 & 0 & $2\left(\Delta_{L}+\bar{\Delta}_{L}\right)$ \\
\hline$c c^{*}$ & 1 & 1 & $\Delta_{R}$ \\
& 0 & 0 & - \\
\hline$b c$ & 3 & -1 & $h+2 \bar{h}$ \\
\hline$b c^{*}$ & 2 & 0 & $h^{\prime}+\bar{h}^{\prime}$ \\
\hline
\end{tabular}

Focusing on model $\mathbf{A}$, as can be observed, the $a c$ and $a c^{*}$ sectors provide exactly three chiral representations $(\overline{4}, 1,2)$ accommodating the right-handed fields $\bar{F}_{R}$ of the three known families and the Higgs pair $H+\bar{H}=$ $(4,2,1)+(\overline{4}, 2,1)$. On the contrary, the $a b$ and $a b^{*}$ sectors, in addition to the three chiral left-handed components $F_{L}=(4,2,1)$, predict four vectorlike representations in $F_{L}+\bar{F}_{L}=(4,2,1)+(\overline{4}, 2,1)$. Similarly, the multiplicities of the remaining representations can be read off from the remaining entries of $\mathrm{Eq}(2)$. Model $\mathbf{B}$, on the other hand, has three chiral representations $F_{L}+\bar{F}_{R}$ and just one vector pair $F_{L}+\bar{F}_{L}$. It can be readily checked that in both models $\operatorname{Tr} Q_{L}$ and $\operatorname{Tr} Q_{R}$ are nonzero, and the spectrum implies anomalies for the corresponding $U(1)_{L}$ and $U(1)_{R}$ factors. As already noted, these anomalies are canceled when VEVs are assigned to appropriate fields. The D-flatness conditions, in particular, read

$$
\begin{aligned}
& \sum_{j} Q_{j}^{(4)}\left|\Phi_{j}\right|^{2}=8\left(|\langle H\rangle|^{2}-|\langle\bar{H}\rangle|^{2}\right)=0 \\
& \sum_{j} Q_{j}^{(L)}\left|\Phi_{j}\right|^{2}=2\left(n_{1}\left|\left\langle\tilde{\nu}_{S}\right\rangle\right|^{2}-n_{2}\left|\left\langle\tilde{\bar{\nu}}_{S}\right\rangle\right|^{2}\right)=-\frac{\operatorname{Tr} Q_{L} g_{s}^{2}}{192 \pi^{2}} M_{S}^{2},
\end{aligned}
$$

$$
\begin{aligned}
\sum_{j} Q_{j}^{(R)}\left|\Phi_{j}\right|^{2}= & 8\left(|\langle H\rangle|^{2}-|\langle\bar{H}\rangle|^{2}\right) \\
& +6\left(m_{1}\left|\left\langle\Delta_{R}\right\rangle\right|^{2}-m_{2}\left|\left\langle\bar{\Delta}_{R}\right\rangle\right|^{2}\right) \\
& +2\left(p_{1}|\langle N\rangle|^{2}-p_{2}|\langle\bar{N}\rangle|^{2}\right) \\
= & -\frac{\operatorname{Tr} Q_{R} g_{s}^{2}}{192 \pi^{2}} M_{S}^{2} .
\end{aligned}
$$

Model B

\begin{tabular}{||lrrl||}
\hline Sector & $\#$ & chirality & Spectrum \\
\hline \hline$a b$ & 0 & 0 & none \\
\hline$a b^{*}$ & 5 & 3 & $4 F_{L}+\bar{F}_{L}$ \\
\hline$a c$ & 3 & -3 & $3 \bar{F}_{R}$ \\
\hline$a c^{*}$ & 4 & 0 & $2(H+\bar{H})$ \\
\hline$a a^{*}$ & 8 & 0 & $4\left(S_{10}+\bar{S}_{10}\right)$ \\
& 3 & 3 & $3 D_{6}$ \\
\hline$b b^{*}$ & 3 & 3 & $3 \bar{\nu}_{s}$ \\
\hline$c c^{*}$ & 5 & 3 & $4 \Delta_{R}+\bar{\Delta}_{R}$ \\
& 6 & 0 & $3(N+\bar{N})$ \\
\hline$b c$ & 6 & 0 & $3(h+\bar{h})$ \\
\hline$b c^{*}$ & 3 & -3 & $3 h^{\prime}$ \\
\hline
\end{tabular}

In the above expressions, the integers $n_{i}, m_{i}, p_{i}$ count the number of the corresponding fields that are assumed to acquire nonzero VEVs. For example, $\max \left(n_{1}, n_{2}\right)=$ $(3,2)$ for model $\mathbf{A}$, and so on. Furthermore, $g_{s}, M_{S}$ are the string coupling and the string scale, respectively.

From this general form of the equations, some important conclusions can be drawn. We first notice that Eq (6) is satisfied for $|\langle H\rangle|=|\langle\bar{H}\rangle|$, i.e., when these two VEVs of the Higgs fields are equal in magnitude. The remaining Dflatness conditions determine the VEVs of the other fields and, consequently, the symmetry breaking scales associated with each one of them. From condition (7), we infer that the VEVs of $V_{s} \sim\left\langle\tilde{\nu}_{s}\right\rangle$ and $\left\langle\tilde{\bar{\nu}}_{s}\right\rangle$ are related to the string scale $\left(V_{s} \leq 10^{-2} M_{S}\right)$. Similarly, Eq. (8) can be solved when either or both kinds of the fields $\Delta_{R}, \bar{\Delta}_{R}, N, \bar{N}$ acquire VEVs of order $V_{s}$. We conclude that in these particular models, the string scale determines either the magnitude of the triplet VEV, and consequently the $S U(2)_{R}$ breaking scale through $\left\langle\Delta_{R}\right\rangle,\left\langle\bar{\Delta}_{R}\right\rangle \neq 0$, or $U(1)_{R}$ whenever $\langle N\rangle,\langle\bar{N}\rangle \neq 0$. If, of course, a string spectrum is obtained where $\operatorname{Tr} Q_{R}=0$, then these VEVs are no longer related to the string scale, and their magnitude can be determined at will. Contemplating the existence of this third class of models in the vast string landscape, in our phenomenological approach that follows, we will assume this third option and comment on alternative possibilities whenever necessary.

In the following, we describe the embedding of the Standard Model states in the 4-2-2 representations and the Higgs mechanism for a two-stage symmetry breaking to the SM. The fermion generations are accommodated in the following representations 


$$
F_{L}+\bar{F}_{R}=(4,2,1)+(\overline{4}, 1,2)
$$

which make up just the 16 of the $S O(10)$. The Standard Model particle assignment is

$$
\begin{aligned}
F_{L}=(4,2,1)= & Q\left(3,2, \frac{1}{6}\right)+\ell\left(1,2,-\frac{1}{2}\right), \\
\bar{F}_{R}=(\overline{4}, 1,2)= & u^{c}\left(\overline{3}, 1,-\frac{2}{3}\right)+d^{c}\left(\overline{3}, 1, \frac{1}{3}\right) \\
& +\nu^{c}(1,1,0)+e^{c}(1,1,1) .
\end{aligned}
$$

The Higgs sector comprises the following fields. The nontrivial representations are the $S U(4)_{C}$ adjoint Higgs field $\Sigma=(15,1,1)$, the bidoublets $h, h^{\prime}$ in $(1,2,2)+$ c.c., and the two Higgs fields $\bar{H}$ and $H$, namely

$$
\begin{aligned}
& \bar{H}=(\overline{4}, 1,2)=\left(\bar{Q}_{H}+\bar{L}_{H}\right) \rightarrow\left(u_{H}^{c}, d_{H}^{c}, e_{H}^{c}, \nu_{H}^{c}\right) \\
& H=(4,1,2)=\left(Q_{H}+L_{H}\right) \rightarrow\left(\bar{u}_{H}^{c}, \bar{d}_{H}^{c}, \bar{e}_{H}^{c}, \bar{\nu}_{H}^{c}\right) .
\end{aligned}
$$

In an $S O(10)$ embedding, we note that $\bar{H}, H$ descend from the 16 and $\overline{16}$ of $S O(10)$, respectively. In addition, a suitable set of singlet fields develop VEVs to provide masses for the vectorlike pairs and right-handed neutrinos. The minimal spectrum derived from the D-brane scenario is given in Table I, where the transformation properties under the various quantum numbers are also shown. Notice, in particular, that the triplet pair $D_{3}^{-}+\bar{D}_{3}^{+}$found in the decomposition of the sextets has the quantum numbers of leptoquarks, since they simultaneously carry both baryon and lepton numbers under the definitions (3), (4).

\section{TWO-STEP SPONTANEOUS SYMMETRY BREAKING}

Having defined the spectrum of the model, in this section we proceed with the implementation of the two-step spontaneous breaking of 4-2-2 symmetry. The $S U(4)_{C} \rightarrow$ $S U(3)_{C} \times U(1)_{B-L}$ breaking is realized with the Higgs field $\Sigma=(15,1,1)$ listed in Table I:

$$
(15,1,1) \rightarrow\langle\Sigma\rangle=\left(\begin{array}{cccc}
\frac{V}{3} & 0 & 0 & 0 \\
0 & \frac{V}{3} & 0 & 0 \\
0 & 0 & \frac{V}{3} & 0 \\
0 & 0 & 0 & -V
\end{array}\right)
$$

Then, at this stage, the symmetry of the model is

$$
S U(3)_{C} \times S U(2)_{L} \times S U(2)_{R} \times U(1)_{B-L} \times U(1)_{L} \times U(1)_{R}
$$

The decomposition of the various representations of the model are shown in Table II.
TABLE II. Spectrum of the left-right symmetric model after the breaking of the 4-2-2 symmetry.

\begin{tabular}{lrrrrr}
\hline \hline$S U(3)_{C} \times S U(2)_{L} \times S U(2)_{R}$ & $Q_{B-L}$ & $Q_{2 L}$ & $Q_{2 R}$ & \multicolumn{1}{c}{$\mathcal{B}$} & $\mathcal{L}$ \\
\hline $3 \times Q_{L}(3,2,1)$ & $\frac{1}{3}$ & -1 & 0 & $\frac{1}{3}$ & 0 \\
$3 \times L(1,2,1)$ & -1 & -1 & 0 & 0 & 1 \\
$3 \times Q_{R}^{c}(\overline{3}, 1,2)$ & $-\frac{1}{3}$ & 0 & 1 & $-\frac{1}{3}$ & 0 \\
$3 \times L_{R}^{c}(1,1,2)$ & 1 & 0 & 1 & 0 & -1 \\
$Q_{H}(3,1,2)$ & $\frac{1}{3}$ & 0 & 1 & $\frac{1}{3}$ & 0 \\
$L_{H}(1,1,2)$ & -1 & 0 & -1 & 0 & 1 \\
$\bar{Q}_{H}(\overline{3}, 1,2)$ & $-\frac{1}{3}$ & 0 & -1 & $-\frac{1}{3}$ & 0 \\
$\bar{L}_{H}(1,1,2)$ & 1 & 0 & 1 & 0 & -1 \\
$D_{3}^{+}(3,1,1)$ & $\frac{2}{3}$ & 0 & 0 & $\frac{2}{3}$ & 0 \\
$\bar{D}_{3}^{+}(\overline{3}, 1,1)$ & $\frac{2}{3}$ & 0 & 0 & $\frac{1}{3}$ & 1 \\
$D_{3}^{-}(3,1,1)$ & $-\frac{2}{3}$ & 0 & 0 & $-\frac{1}{3}$ & -1 \\
$\bar{D}_{3}^{-}(\overline{3}, 1,1)$ & $-\frac{2}{3}$ & 0 & 0 & $-\frac{2}{3}$ & 0 \\
$\Delta_{R}(1,1,3)$ & 0 & 0 & \pm 2 & $\cdots$ & $\cdots$ \\
$(N, \bar{N})$ & 0 & 0 & \pm 2 & $\cdots$ & $\cdots$ \\
$\Delta_{L}(1,3,1)$ & 0 & \pm 2 & 0 & $\cdots$ & $\cdots$ \\
$\left(\nu_{s}, \bar{\nu}_{s}\right)$ & 0 & \pm 2 & 0 & $\cdots$ & $\cdots$ \\
$h, h^{\prime}(1,2,2)$ & 0 & \pm 1 & $\mp 1$ & $\cdots$ & $\cdots$ \\
\hline \hline
\end{tabular}

The breaking of the left-right symmetric group in Eq. (14) to the SM gauge group takes place from the nonzero VEVs along the neutral components $\left\langle\nu_{H}^{c}\right\rangle,\left\langle\bar{\nu}_{H}^{c}\right\rangle$ of the right-handed doublet fields $L_{H}, \bar{L}_{H}$ in $H, \bar{H}$. Notice that, these Higgs fields are also charged under $U(1)_{R}$. Then, their VEVs, combined with the $\bar{N}, \nu_{s}$ singlet nonzero VEVs, which will be discussed later, will also break the $U(1)_{L}$ and $U(1)_{R}$ symmetries. At the final stage, the SM symmetry breaking occurs with a nonzero VEV of the bidoublet $h$.

Before studying some implications for the effective low energy theory, recall first that in realistic D-brane constructions, such as those presented in Eq (5), in addition to the chiral states $F_{L}, \bar{F}_{R}$ accommodating the fermion generations, vectorlike pairs such as $F_{L}^{i}+\bar{F}_{L}^{i}, F_{R}^{i}+\bar{F}_{R}^{i}, D_{6}^{i}+\bar{D}_{6}^{i}$, $i=1,2, \ldots$ etc., are also present in the zero mode spectrum. However, such pairs arise with opposite $U(1)$ charges and, in principle, receive heavy masses of the order of the GUT scale, namely $\mathcal{W} \supset M_{\mathrm{GUT}}\left(F_{L}^{i} \bar{F}_{L}^{i}+F_{R}^{i} \bar{F}_{R}^{i}+D_{6}^{i} \bar{D}_{6}^{i}\right)$. For the Higgs fields given in Eqs. (11) and (12), however, there is an additional contribution due to the coupling with the adjoint,

$$
\mathcal{W}_{H} \supset \bar{H} \Sigma H+M_{H} \bar{H} H
$$

Substituting Eq. (13), we obtain

$$
\mathcal{W}_{H} \supset\left(\frac{V}{3}+M_{H}\right) \bar{Q}_{H}^{c} Q_{H}^{c}+\left(M_{H}-V\right) \bar{L}_{H} L_{H},
$$

where $L_{H}^{c}=\left(e_{H}^{c}, \nu_{H}^{c}\right)^{T}, Q_{H}^{c}=\left(u_{H}^{c}, d_{H}^{c}\right)^{T}$ are $S U(2)_{R}$ doublets, and $\bar{L}_{H}^{c}, \bar{Q}_{H}^{c}$ their complex conjugates. In the two-step 
breaking pattern, we anticipate that the $S U(2)_{R}$ symmetry breaking scale $M_{R}$, determined by the $L_{H}^{c}, \bar{L}_{H}^{c}$ VEVs, is of the order of a few $\mathrm{TeV}$. Therefore, choosing $V \simeq M_{H} \sim \mathcal{O}\left(M_{\mathrm{GUT}}\right), L_{H}^{c}, L_{H}^{c}$ remain in the low energy spectrum while $\bar{Q}_{H}^{c} Q_{H}^{c}$ acquire masses $M_{Q_{H}} \sim \frac{4}{3} M_{H} \sim$ $\mathcal{O}\left(M_{\mathrm{GUT}}\right)$. This "singlet-triplet" splitting is similar to the "doublet-triplet" splitting in the $S U(5)$ scenario.

In accordance with our discussion around Eq. (5), the relation of the two scales $M_{\mathrm{GUT}}$ and $M_{R}$ depends on the specifics of the particular string construction. Hence, while $M_{\mathrm{GUT}}$ is naturally close to the compactification scale, $M_{R}$ depends on the $U(1)_{R}$ properties of the spectrum of the particular string model. More precisely, if $\operatorname{Tr} Q_{R} \neq 0$ in Eq. (8), then $M_{R}$ cannot be many orders of magnitude smaller than $M_{\mathrm{GUT}}$. In this case, the requirement of a few TeV scale $M_{R}$ could be realized only in low-scale string models. Notice that in the context of D-brane scenarios this is a viable possibility. Indeed, one of the main obstacles in low energy unified models is the problem of gauge coupling unification. However, this can be evaded since in the 4-2-2 D-brane model there is no compelling reason that the couplings should unify at the GUT scale. As we have mentioned, a second possibility may also occur, where $\operatorname{Tr} Q_{R}=\operatorname{Tr} Q_{R}^{3}=0$. In this case, $M_{R}$ disentangles from the compactification scale and $S U(2)_{R}$ can be fixed by arranging the $S U(R)_{R}$ breaking VEVs according to the phenomenological requirements. In the following, we will investigate the implications of this case.

\section{LOW ENERGY PHENOMENOLOGY}

In the previous sections we stressed that there is a natural way to provide masses for the vectorlike states that might appear in the spectrum. Hence, fields such as color sextets $D_{6}$ and triplets $\Delta_{L, R}$ of Table I decouple from the light spectrum as long as they appear in pairs with opposite $U(1)$ charges. We also noticed that, when the $S U(4)_{C}$ adjoint Higgs acquires its VEV, the Higgs pair $H+\bar{H}$ is a remarkable exception to this rule and, therefore, it remains in the "massless" spectrum of the effective theory.

In this section, we analyze the Yukawa sector and, in particular, the terms generating masses for the SM charged fields and neutrinos. The superpotential terms of the effective model should be invariant under the gauge symmetry of the original D-brane configuration of the model. We will also introduce "matter" parity $\mathcal{R}$ to suppress unwanted superpotential terms and possible exotic interactions. To this end, the fields $\Sigma, H, \bar{H}, \bar{N}, h$ acquire VEVs and will be assigned positive $\mathcal{R}$ parity, and the fields $F_{L}, \bar{F}_{R}, N$ are assigned negative $\mathcal{R}$ parity.

Next, we analyze the contributions of the tree level terms. The third family charged fermion and Dirac neutrino mass could emerge, to a good approximation, from a common Yukawa term, $\lambda \bar{F}_{R} F_{L} h$. As a result, in the simplest constructions, the 4-2-2 symmetry imposes approximate third family Yukawa unification $\lambda_{t} \simeq \lambda_{b} \simeq \lambda_{\tau} \simeq \lambda_{\nu_{D}}$.

However, these are not the only mass terms for the neutrino sector. The following invariant couplings also involve the neutrino fields

$$
\lambda \bar{F}_{R} F_{L} h+\lambda_{1} \bar{F}_{R} H N+\lambda_{2} \frac{1}{M_{*}} \bar{N}^{2} N N,
$$

where $M_{*}$ is the compactification scale, $M_{*} \gtrsim M_{\mathrm{GUT}}$. The term $\bar{F}_{R} H N$ suggests that the singlet field $N=$ $(1,1,1)_{(0,0,-2)}$ can be interpreted as a sterile neutrino. Therefore, assuming a nonzero VEV for the scalar component of the $\bar{N}$ singlet (positively charged under $U(1)_{R}$ ), Eq. (17) yields

$$
\begin{aligned}
\mathcal{W} & =\lambda_{1}\left\langle h_{u}\right\rangle \nu^{c} \nu+\lambda_{2}\langle H\rangle \nu^{c} N+\frac{\lambda_{3}}{M_{*}}\langle\tilde{\bar{N}}\rangle^{2} N N \\
& =m_{D} \nu^{c} \nu+V_{R} \nu^{c} N+\mu N N
\end{aligned}
$$

where we have defined

$m_{D}=\lambda_{1}\left\langle h_{u}\right\rangle, \quad V_{R}=\lambda_{2}\langle H\rangle, \quad \mu=\frac{\lambda_{3}}{M_{*}}\langle\tilde{\bar{N}}\rangle^{2}$.

The new neutral state $N$, coupled to the ordinary neutrino fields with the superpotential couplings (17), gives rise to an inverse seesaw mechanism $[18,19]$. Assuming three $N$ singlet fields, in particular, we obtain a $6 \times 6$ matrix of the form

$$
M_{\nu} \sim\left(\begin{array}{lll}
0 & m_{D}^{T} & 0 \\
m_{D} & 0 & V_{R} \\
0 & V_{R} & \mu
\end{array}\right) .
$$

The three left-handed neutrinos obtain eigenmasses of order

$$
m_{\nu} \approx \frac{m_{D}^{2}}{V_{R}^{2}} \mu
$$

which must be sufficiently light (of order $10^{-1}-10^{-2} \mathrm{eV}$ ) to interpret the neutrino oscillations data.

In order to provide an estimate, we make the following assumptions for the various scales involved in Eq. (21). Observing that the singlet $\bar{N}$ carries $U(1)_{R}$ charge, we first make the reasonable assumption that its $\mathrm{VEV}$ is associated with the $S U(2)_{R}$ breaking scale

$$
\langle\bar{N}\rangle=\kappa V_{R} \sim \mathcal{O}\left(V_{R}\right),
$$

where $\kappa$ is a dimensionless constant. Then, from Eq. (19), we find that the scale $\mu$ is 


$$
\mu=\kappa^{2} \frac{V_{R}^{2}}{M_{*}}
$$

Substituting into Eq. (21), we find

$$
m_{\nu} \simeq \kappa^{2} \frac{m_{D}^{2}}{M_{*}}
$$

For $M_{*}$ of the order of the GUT scale and the Dirac mass $m_{D}$ of the electroweak mass scale, and with $\kappa \gtrsim 1$, we can obtain the desired left-handed neutrino masses.

The interesting fact here is that a new mass scale $\mu$ is effectively generated, and it is associated with the "sterile neutrino" $N$. Since the light neutrino scale computed from the inverse seesaw matrix does not depend on the details of the $S U(2)_{R}$ breaking scale, we are free to choose $V_{R}$ (provided it satisfies the experimental bounds) so that the emergent scale $\mu$ takes the desired value. Thus, a well motivated choice is related to the possible interpretation of the dark matter puzzle through the existence of a light (order a few keV) sterile neutrino. ${ }^{2}$ In general, the two eigenvalues of this matrix are almost degenerate. However, with the particular choice of the $S U(2)_{R}$ breaking scale and the existence of several neutral singlets, as is the case in the present D-brane construction, a light neutral state can emerge naturally. ${ }^{3}$

As an example, assuming that the $\mu$ parameter in Eq. (20) represents a submatrix of the extra singlets with a scale of the order $10 \mathrm{keV}$, the scale $V_{R}$ is given by

$$
V_{R} \sim \sqrt{M_{*} / \mathrm{GeV}} 10^{-3} \mathrm{GeV} \gtrsim 10^{6} \mathrm{GeV} .
$$

Working out the eigenvalues of the mass matrix (20), one can see that it is plausible to obtain a light neutral state of mass $\mathcal{O}(10 \mathrm{keV})$ that could be interpreted as a dark matter component, provided that it is sufficiently long lived.

A major issue in many grand unified models is the problem of rapid proton decay. The 4-2-2 symmetry does not contain the gauge bosons that mediate dimension six baryon number violating processes. Then, the only source of proton decay is associated with dimension five or higher dimensional operators, related to graphs containing SM color triplets and other states, and the predictions are very model dependent. Operators of this kind are of the form $Q Q Q \ell, Q Q u^{c} e^{c}, u^{c} d^{c} d^{c} \nu^{c}$, etc., and may arise from nonrenormalizable terms of the form $F_{L} F_{L} F_{L} F_{L}, F_{L} F_{L} \bar{F}_{R} \bar{F}_{R}$, and $\bar{F}_{R} \bar{F}_{R} \bar{F}_{R} \bar{F}_{R}$. Such couplings, however, are prevented from the additional

\footnotetext{
${ }^{2}$ See, e.g., Refs. [20,21] and references therein. Also, our estimates are in agreement with recent constraints [22] arising from the Galactic Center give a bound $m_{\nu_{s}} \leq 15 \mathrm{keV}$.

${ }^{3}$ See Refs. [23,24] for recent related work.
}

Abelian symmetries $U(1)_{L, R}$ in the present construction. Then, the only available states that might mediate baryon violating diagrams are the triplets descending from the sextets, which, in principle, receive masses of order $M_{\text {GUT }}$. Nonrenormalizable Yukawa couplings of the form $\frac{1}{M_{\text {str }}} F_{L} F_{L} D_{6} \nu_{s}$ and $\frac{1}{M_{\text {str }}} \bar{F}_{R} \bar{F}_{R} \bar{D}_{6} N$ give rise to diagrams similar to those discussed in Ref. [25], and their strength is determined by the VEV of the scalar component of the singlet $\nu_{s}$. There are no constraints on the possible values of $\left\langle\tilde{\nu}_{s}\right\rangle$, but if this is taken to be close to the GUT scale, the proton lifetime is estimated to be of order $10^{35}-10^{36}$ yrs., which will be tested by the recently approved Hyper Kamiokande experiment [26].

\section{DISCUSSION AND SUMMARY}

In this paper, we have analyzed a class of 4-2-2 models within the framework of intersecting D-branes. The low energy spectrum contains additional particles that normally do not appear in the usual field theory based 4-2-2 models. We focused on breaking the 4-2-2 symmetry to the SM gauge group via an intermediate step $S U(3)_{C} \times S U(2)_{L} \times S U(2)_{R} \times U(1)_{B-L}$. The first stage of the two-step breaking pattern is realized with the $S U(4)$ adjoint VEV, which does not transform under the $S U(2)_{L / R}$, and so these latter gauge symmetry factors are preserved. The second stage of symmetry breaking proceeds with the use of the $H+\bar{H}=\left(Q_{H}+L_{H}\right)+\left(\bar{Q}_{H}^{c}+\right.$ $\left.\bar{L}_{H}^{c}\right)$ representations of the 4-2-2 group. Implementing a missing partner mechanism discussed in the text, the righthanded doublets $\bar{L}_{H}^{c}+L_{H}^{c}$ remain in the low energy spectrum and develop TeV scale VEVs along their neutral directions $\left\langle\nu_{H}^{c}\right\rangle$ and $\left\langle\bar{\nu}_{H}^{c}\right\rangle$ respectively, thereby breaking the $S U(2)_{R}$ symmetry.

The possibility of $\mathrm{keV}$ mass sterile neutrino as a potential new dark matter candidate is a striking example of this. The appearance of additional $U(1)$ symmetries that prevent rapid proton decay is certainly very helpful and a much desired feature for realistic model building. Proton decay via higher dimensional operators can yield lifetime estimates on the order of $10^{35}-10^{36}$ yrs., which will be tested by the Hyper Kamiokande experiment.

The prospects for the embedding of this scenario in a fully fledged D-brane construction has also been discussed and will be the subject of future investigation.

\section{ACKNOWLEDGMENTS}

G. K. L. would like to thank the Physics and Astronomy Department and Bartol Research Institute of the University of Delaware, for kind hospitality where part of this work has been done. Q. S. is supported in part by the DOE Grant No. DE-SC0013880. 
[1] J. C. Pati and A. Salam, Phys. Rev. D 10, 275 (1974); 11, 703(E) (1975).

[2] A. M. Sirunyan et al. (CMS Collaboration), Phys. Rev. D 97, 072006 (2018).

[3] G. Aad et al. (ATLAS Collaboration), Phys. Lett. B 754, 302 (2016).

[4] R. N. Mohapatra and J. C. Pati, Phys. Rev. D 11, 566 (1975).

[5] Q. Shafi and C. Wetterich, Phys. Lett. B 73, 65 (1978).

[6] R. N. Mohapatra and G. Senjanovic, Phys. Rev. Lett. 44, 912 (1980); G. Senjanovic and V. Tello, Proc. Sci. PLANCK2015 (2016) 141.

[7] G. Lazarides, Q. Shafi, and C. Wetterich, Nucl. Phys. B181, 287 (1981).

[8] J. C. Pati, Int. J. Mod. Phys. A 32, 1741013 (2017).

[9] CMS Collaboration, CERN Report No. CMS-PAS-B2G-16023, 2018; G. Aad et al. (ATLAS Collaboration), J. High Energy Phys. 12 (2015) 055.

[10] I. Antoniadis and G. K. Leontaris, Phys. Lett. B 216, 333 (1989).

[11] G. K. Leontaris and J. Rizos, Phys. Lett. B 510, 295 (2001).

[12] M. Cvetic, T. Li, and T. Liu, Nucl. Phys. B698, 163 (2004).

[13] M. Cvetic, D. Klevers, D. K. M. Peña, P. K. Oehlmann, and J. Reuter, J. High Energy Phys. 08 (2015) 087.

[14] L. E. Ibanez, F. Marchesano, and R. Rabadan, J. High Energy Phys. 11 (2001) 002.
[15] P. Anastasopoulos, G. K. Leontaris, and N. D. Vlachos, J. High Energy Phys. 05 (2010) 011.

[16] P. Anastasopoulos, T. P. T. Dijkstra, E. Kiritsis, and A. N. Schellekens, Nucl. Phys. B759, 83 (2006).

[17] T. P. T. Dijkstra, L. R. Huiszoon, and A. N. Schellekens, Nucl. Phys. B710, 3 (2005).

[18] R. N. Mohapatra and J. W. F. Valle, Phys. Rev. D 34, 1642 (1986).

[19] M. Malinsky, J. C. Romao, and J. W. F. Valle, Phys. Rev. Lett. 95, 161801 (2005).

[20] S. Dodelson and L. M. Widrow, Phys. Rev. Lett. 72, 17 (1994).

[21] A. Merle, V. Niro, and D. Schmidt, J. Cosmol. Astropart. Phys. 03 (2014) 028.

[22] R. Yunis, C. R. Argüelles, N. E. Mavromatos, A. Moliné, A. Krut, J. A. Rueda, and R. Ruffini, arXiv:1810.05756.

[23] K. Agashe, P. Du, M. Ekhterachian, C. S. Fong, S. Hong, and L. Vecchi, Phys. Lett. B 785, 489 (2018).

[24] V. Brdar and A. Y. Smirnov, J. High Energy Phys. 02 (2019) 045.

[25] G. K. Leontaris and Q. Shafi, Phys. Rev. D 96, 066023 (2017).

[26] K. Abe et al. (Hyper-Kamiokande Collaboration), Prog. Theor. Exp. Phys. 2018, 063 C01 (2018). 